\title{
Cell Growth in Response to Mechanical Stiffness is Affected by Neuron- Astroglia Interactions
}

\author{
Xue Jiang ${ }^{\#, 2}$, Penelope C. Georges ${ }^{\#, 1,2}$, Baogang $\mathrm{Li}^{\#, 1}$, Yangzhou $\mathrm{Du}^{1}$, Melinda K. Kutzing ${ }^{2,4}$, \\ Michelle L. Previtera ${ }^{1,3}$, Noshir A. Langrana ${ }^{2}$, Bonnie L. Firestein*, \\ ${ }^{1}$ Department of Cell Biology and Neuroscience and ${ }^{2}$ Department of Biomedical Engineering, ${ }^{3}$ Molecular Biosciences \\ Graduate Program, ${ }^{4}$ Biomedical Engineering Graduate Program, Rutgers University, Piscataway, New Jersey 08854, USA
}

\begin{abstract}
Cell adhesion and morphology are affected by the mechanical properties of the extracellular matrix. Using polyacrylamide gels as cell substrates, the cellular response to substrate compliance was investigated in pure neuronal, pure astroglial, or mixed co-cultures. Substrates used spanned a large range of stiffnesses including that of brain tissue. In both pure and mixed cultures, immature (vimentin+) astroglia adhered best to stiffest gels. Mature (GFAP+) astrocyte adhesion peaked on intermediate stiffness, while pure GFAP+ astroglial adhesion displayed no intermediate preference and increased with stiffness. Neurite length was constant with stiffness; however, primary dendrite number was lowest on intermediate gels. Pure neuronal cultures were more adherent to hard gels, while mixed cultures had no stiffness preference. Furthermore, we investigated the role of stiffness in the modulation of the neurotoxic effect of glutamate. Exposure to two glutamate concentrations $(500$ and $1000 \mu \mathrm{M})$ of cultured spinal cord neurons induced cell death. The damage elicited by $500 \mu \mathrm{m}$ glutamate to neurons in a mixed culture of spinal cord cells is most severe on soft $300 \mathrm{~Pa}$ gels. The neurotoxic effect of glutamate on neurons cultured on hard gels where astrocytes are present was strongly attenuated compared with that observed on soft gels, where there is a relatively low number of astrocytes. Our data suggest that mechanical stiffness of the substrate affects the response of both neurons and astroglia, and this response is varied by interaction between the two cell types.
\end{abstract}

\section{INTRODUCTION}

Cells can integrate and respond to biological and chemical information as well as the physical properties of their extracellular matrix (ECM) [1-3]. Among critical extracellular cues, the effects of physical stimuli, including traction force, mechanical strain, surface topography, and in particular, substrate stiffness, have been identified and found to affect cellular behavior in a cell-type specific manner [4-7]. Wang and co-workers popularized bis-acrylamide crosslinked hydrogels ('bis-gels', hereafter) as substrates to study cell-substrate interaction [8]. This model system and protocol has been utilized for a variety of cell types, including mouse spinal cord and rat cortical neurons $[9,10]$; Table 1 shows a partial list of previous studies utilizing this gel system. Currently, there are few studies on neuronal cell types, and fewer still on cells of the central nervous system (CNS). Mouse spinal cord neurons have more dendritic branches on softer gels $(\mathrm{E} \sim 150 \mathrm{~Pa})$ than on stiffer gels $(\mathrm{E} \sim 1500 \mathrm{~Pa})$, and glial cells do not grow on these gels. Rat cortical astrocytes spread less and adhere poorly to softer gels with a shear modulus of $200 \mathrm{~Pa}(\mathrm{E} \sim 600 \mathrm{~Pa})$, while rat cortical neurons co-cultured with glia extend neurites and adhere equally on soft and hard gels $(\mathrm{E} 27 \mathrm{kPa})$.

It is not yet clear how astroglia and neurons affect each other's growth on biomaterials of different stiffnesses. This topic is especially relevant in applications where a differential response to stiffness changes by neurons and astroglia is desired. For instance, scaffolds that supply mechanical cues

*Address correspondence to this author at the Department of Cell Biology and Neuroscience, Rutgers University, Piscataway, NJ 08854, USA; E-mail: firestein@biology.rutgers.edu

"These authors contributed equally to this mauscript. in order to inhibit astroglia growth and promote neurite regeneration could be used to bypass the scar formation following spinal cord injuries.

Until now, the majority of previous studies have been performed on bis-gel stiffnesses lower than $80 \mathrm{kPa}$ (Table 1). Published results describing the mechanical properties of CNS tissue derived from different species and subjected to different testing schemes report a broad range of stiffnesses, from a few $\mathrm{Pa}$ to hundreds of $\mathrm{kPa}$ [14-16]. Furthermore, many polymers, such as pHEMA (poly-hydroxy-ethyl methacrylate) and PLGA (poly-lactic-co-glycolic acid), used as implant materials in CNS tissue repair, have mechanical stiffnesses in the $100 \mathrm{kPA}$ to MPa range [17, 18]. Thus, from an engineering perspective, it is important to examine cellular behavior on higher stiffnesses than those previously examined. Additionally, though achieving a resemblance to the natural cell environment (i.e. fluidic and soft ECM) when designing biomaterials is important, it is not without benefits to examine other possibilities (e.g., high stiffness), considering good neuronal growth on plastic culture plates that have a stiffness of GPa magnitude.

Thus, the objective of this study is to investigate spinal cord cell adhesion and morphology on bis-gels whose mechanical properties span a large range, varying from $\sim 300 \mathrm{~Pa}$ (soft gel) to $\sim 230 \mathrm{kPa}$ (hard gel) and to elucidate the role of neuron-astroglia interactions in mechano-sensing by neurons and glia. Here, we evaluate the number of adherent cells and the morphological properties of spinal cord neurons and astroglia in mixed and pure cultures. Furthermore, we examine functional aspects of our co-culture system by studying the effect of matrix stiffness on glutamate toxicity. Results from this study will contribute to our understanding of the effects 
Table 1.

\begin{tabular}{|c|c|c|c|}
\hline Cell Type & \multicolumn{2}{|c|}{ Stiffness (Pa) } & Major Observations \\
\hline & Low & High & $\downarrow$ Cells spreading and $\uparrow$ migration rate on soft gels; [11] \\
\hline \hline Rat kidney eptithelial/3T3 & 20,000 & 80,000 & Directional migration from soft to stiff area [12] \\
\hline Bovine VSMC & 5,000 & 35,000 & $\downarrow$ Cell compaction, liver-specific albumin secretion and spreading on soft gels; [13] \\
\hline Hepatocyte & 2,000 & 10,000 & $\downarrow$ Glial growth and neuron branch points $\uparrow$ on soft gels [9] \\
\hline Mouse spinal cord & 50 & 550 & $\downarrow$ Astrocyte spreading and organized F-actin on soft gels; = Neurite length and adhesion [10] \\
\hline Cortical cells & 200 & 9,000 & \\
\hline
\end{tabular}

of neuron-astroglia interactions on each cell type's response to mechanical stiffness and guide future design of biomaterials to control both neuronal and astroglial growth as well as neuronal dendrite formation.

\section{MATERIALS AND METHODS}

\section{Gel Mechanical Characterization}

Bis-gels with monomer concentrations of $3 \%$ and $5 \%$ and fixed monomer/bis-crosslink ratio (29:1) were prepared in microcentrifuge tubes as described previously $[19,20]$. A steel bead $(0.79 \mathrm{~mm}$ in diameter; Small Parts, Miami Lakes, FL) was embedded in the gel during polymerization, and a customized fixture was utilized to measure the mechanical properties of the gels (Fig. 1). A magnet was used to apply magnetic forces over the steel bead embedded in the gels in the microcentrifuge tube (shown in insert of Fig. 1). Displacement of the bead under applied magnetic fields is measured with the images captured by video microscope and mechanical properties calculated.

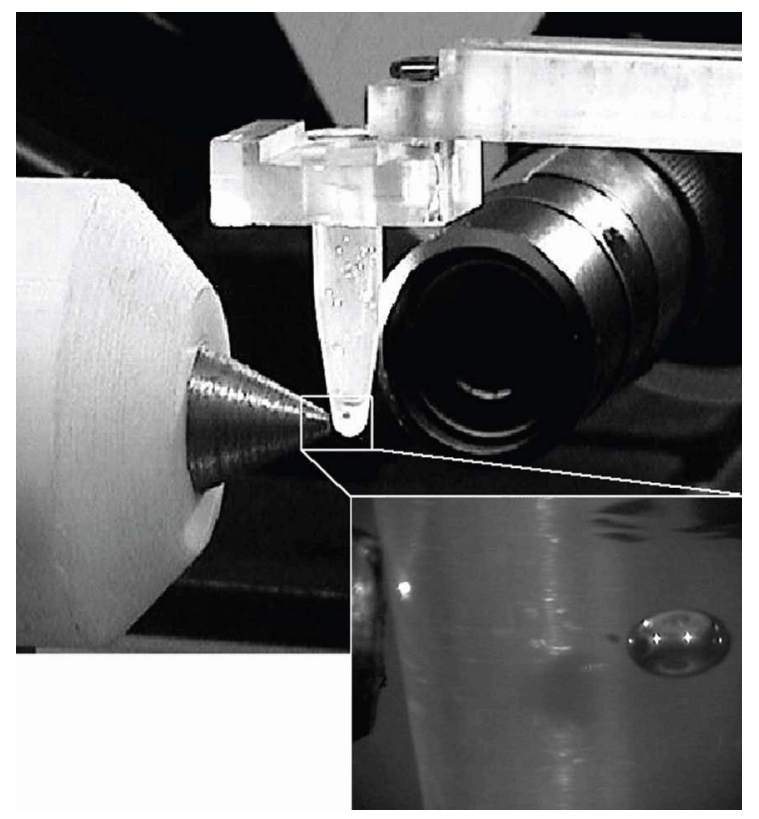

Fig. (1). Experimental setup for non-invasive measurement of mechanical properties of bis-crosslinked polyacrylamide hydrogels.

The bulk elasticity modulus of the hydrogel is a function of the monomer concentration and crosslinker density [19, 20]. A calibrated magnetic force was applied to the bead and the displacement was measured with a video microscope.
The experimental method is non-intrusive and was used to monitor gel compliance changes. Classical linear elasticity theory was used to model the test conditions as a problem of a rigid spherical inclusion in an isotropic, elastic medium. Under the influence of an externally applied known magnetic load $F$, the sphere is displaced by a distance $\delta$, which depends on the elastic modulus, Poisson's ratio, and the boundary conditions. Assuming gels are incompressible as they essentially are, the modulus computed, $E_{a}$, was expressed in the form:

$$
E_{a}=\frac{\rho}{2 \pi R_{\circ}}\left(\frac{\mathrm{F}}{\delta}\right)
$$

where $R_{0}$ is the radius of the sphere and $\rho$ is a geometrical factor to account for the effect of finite boundary. In this study, $\rho$ is determined to be 0.967 .

Stiffnesses of bis-gels with monomer concentration higher than $10 \%$ (including 10\%, 15\% and 20\%) were evaluated by standard tensile tests (sample cross section diameter $15.24 \mathrm{~mm}$, and length varying from 38.1 to $66.0 \mathrm{~mm}$ ). Each test was repeated at least twice.

\section{Bis-Gel Preparation}

Round glass coverslips (12 mm) were used for gel preparation according to the protocol of Pelham and Wang [8]. Briefly, the glass surface was activated by treatment with 3aminopropyltrimethxysilane and $0.5 \%$ glutaraldehyde (Sigma). A series of gels was prepared by pipetting $12 \mu \mathrm{l}$ of gel solutions, containing 3-20\% acrylamide monomer (acrylamide: bis of 29:1, EMD Chemicals Inc., Gibbstown, NJ), $0.1 \%$ ammonium persulfate, $0.1 \%$ TEMED, on the glass coverslip and covering the coverslip with another siliconized glass coverslip. After polymerization was completed, the top coverslip was removed, and the gel bound to the bottom coverslip was transferred into a 24-well culture plate. Gels were washed and functionalized with Sulfo-SANPAH (Pierce, Rockford, IL). The gels were then coated with polyD-lysine (1 $\mathrm{mg} / \mathrm{ml}$ solution in water). Care was taken to maintain flatness of the gel surface in minimizing the effect of surface topography. The average thickness of the prepared gels was approximately $100 \mu \mathrm{m}$.

\section{Mixed Spinal Cord Cell Culture}

Spinal cords were dissected from embryonic day 16 Sprague Dawley rats (Taconic, Hudson, NY), and cells were dissociated and plated on gels in 24-well plates at a cell den- 
sity of 25,000 cells $/ \mathrm{cm}^{2}$. Cells were cultured in serumcontaining medium (SCM; MEM containing $10 \%$ horse serum, $1 \%$ pen/streptomycin, $0.6 \%$ glucose) at $37^{\circ} \mathrm{C}$ for 1 day. At 2 DIV (days in vitro) SCM was changed to Neurobasal (NB) medium (Gibco) containing 2\% B27 supplement (Gibco), $69 \mu \mathrm{g} / \mathrm{ml} \quad$ L-glutamine, $25 \mu \mathrm{M}$ betamercaptoethanol, and $1 \%$ penicillin/streptomycin and grown for 6 days, after which the cells were fixed with $4 \%$ paraformaldehyde in PBS.

\section{Pure Spinal Cord Neuron Cultures}

To grow pure spinal neuron cultures, we followed the same steps in culturing mixed culture except that after changing SCM to NB medium and allowing cells to grow for additional 24 hours, cytosine arabinoside (Ara-C, $5 \mu \mathrm{M}$ ) was added to these cultures. The cell culture was maintained for 3 days after which the Ara-C-containing media was changed to fresh NB media without Ara-C. The neurons were grown for another 3 days and were fixed with $4 \%$ paraformaldehyde in phosphate-buffered saline.

\section{Pure Astroglial Cultures}

Spinal cord astroglial cultures were derived from postnatal day 1 rats using a modified approach [21]. Spinal cords were dissected and dissociated with trituration. Cells were plated in tissue culture flasks at density of 1000 cells $/ \mathrm{mm}^{2}$. Cultures were grown in NM-15 medium (84.4\% MEM, 15\% Fetal Bovine Serum, $0.6 \%$ glucose, supplemented with penicillin/streptomycin) for 9 days. Medium was changed every 3 days. The flasks were then shaken at $400 \mathrm{x}$ rpm for 20 minutes before the medium was changed and the floating cells (mostly microglia) were washed away. The flasks were again shaken overnight at $250 \mathrm{x}$ rpm to remove attached oligodendrocytes and remaining microglia. Following shaking, floating cells were washed away and medium was changed. Ara-C was added to final concentration of $10 \mu \mathrm{M}$ to reduce the number of undifferentiated cells. Cultures were grown for 3 additional days before being trypsinized and re-plated on gels in 24-well plates with a cell density of 25,000 cells $/ \mathrm{cm}^{2}$.

\section{Immunostaining and Cell Counting}

Cells fixed on gels were blocked with PBS containing $2 \%$ normal goat serum, $0.1 \%$ Tween, and $0.02 \%$ sodium azide for $2 \mathrm{hr}$, and incubated with primary antibody overnight at $4^{\circ} \mathrm{C}$. Primary antibodies were as follows: mouse anti-MAP2 antibody to label neurons (1:500, BD PharMingen, San Diego, CA); rabbit anti-GFAP antibody to label mature astrocytes (1:200, Abcam, Cambridge, MA); mouse anti-vimentin antibody to identify immature astrocytes (1:500, Chemicon Inc., Temecula, CA). Cells were washed three times with PBS and incubated with secondary antibodies (Cy3-conjugated anti-mouse IgG (1:200); Cy2conjugated anti-rabbit IgG (1:200, Jackson ImmunoResearch, West Grove, PA)) for $1 \mathrm{hr}$ at RT. DAPI nuclear staining was performed to identify all intact cells in the culture. Cells were observed using fluorescence microscopy (Olympus IX50 microscope with a Cooke Sensicam CCD cooled camera, fluorescence, imaging system and Image Pro software (MediaCybernetics)), and MAP2+, GFAP+, or vimentin+ cells were counted from eight randomly selected areas of each well, aided with the corresponding images of
DAPI staining. Each experiment was repeated at least three times, and there were at least three gel samples for each group in every experiment.

\section{Neurite Number and Length}

Neurons were fixed and immunostained for MAP2 as described above. Primary dendrites were counted as described previously [22]. Neurite length was measured by using Scion Image software (Scion Corp., Frederick, MD). Only those neurites with a clear end point were measured and when the neurite connects two neurons, half of the neurite length was counted for both neurons. Dendrites of those neurons that overlap each other, which prevented origin determination, were not measured.

\section{Glutamate Treatment}

Reagents used to treat cells were made into stock solutions. Glutamate was dissolved in Locke's buffer $(154 \mathrm{mM}$ $\mathrm{NaCl} ; 5.6 \mathrm{mM} \mathrm{KCl} ; 2.3 \mathrm{mM} \mathrm{CaCl}_{2} ; 1.0 \mathrm{mM} \mathrm{MgCl} 2 ; 3.6 \mathrm{mM}$ $\mathrm{NaHCO}_{3} ; 5 \mathrm{mM}$ glucose; $5 \mathrm{mM}$ Hepes; $\mathrm{pH}$ 7.2) and diluted as indicated. Cell medium was replaced with solutions of with or without (control) glutamate in Locke's buffer, for $1 \mathrm{~h}$ at $37^{\circ} \mathrm{C}$, after which the solution was replaced with cell culture medium. In all cases, cells were incubated another $24 \mathrm{~h}$ at $37^{\circ} \mathrm{C}$ before fixation. For each group, pictures were taken for 10 random fields at a magnification of 20X. MAP-2 (+) cell number was counted by the experimenter blinded to the condition. Cell numbers in control and treated groups were compared with one-way analysis of variance (ANOVA) followed by Tukey's tests for multiple comparisons.

\section{RESULTS}

\section{Gel Mechanical Characterization}

The elastic moduli of bis-gels with different monomer concentrations were either computed based on the formula described in the Materials and Methods section with the experimental measurements of applied magnetic forces and

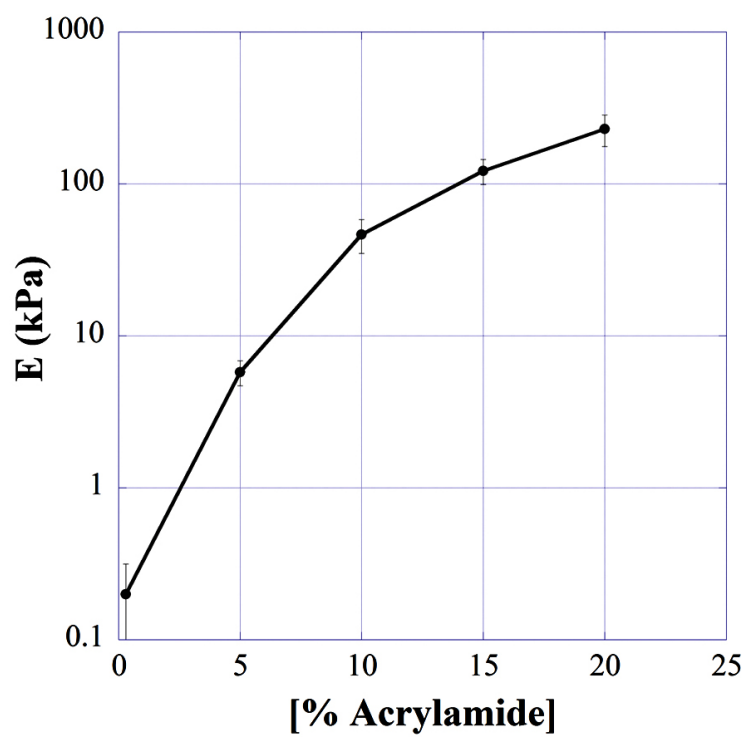

Fig. (2). Stiffness of the polyacrylamide hydrogel with respect to the acrylamide monomer concentration at fixed monomer/crosslink ratio of 29:1. 
displacements, or measured by standard tensile tests (for gels with stiffness over $\sim 50 \mathrm{kPa}$ ). Mechanical stiffness of gels with acrylamide concentration ranging from 3 to $20 \%$ result in Young's moduli that span three orders of magnitude (Fig. 2). Note that mechanical stiffness and elastic modulus were used interchangeably in this work. Cells are mechanosensing the stiffness measured, as it has been previously reported, and also confirmed in this study, that functionalization and protein conjugation do not alter the mechanical stiffness of the gels [9].

\section{Effect of Gel Stiffness Cell Growth in Mixed Cultures}

Typical morphology of neurons, mature (GFAP+) astrocytes, and immature (vimentin+) astrocytes in their coculture on gels of two of five different mechanical stiffnesses is shown in Fig. (3). On all stiffnesses tested, rat spinal cord neurons extended processes from cell bodies, exhibiting no distinct difference from their counterparts on culture plates. Mature astrocytes (GFAP+) assumed a variety of morphologies: an extended string-shape, a star shape or a small, round irregular shape, whereas vimentin+ immature astrocytes displayed only the round (on softest gels) and polygonal morphologies. We also examined the growth of oligodendrocytes on gels by CNPase staining, and we found no detectable CNPase+ cells (data not shown).

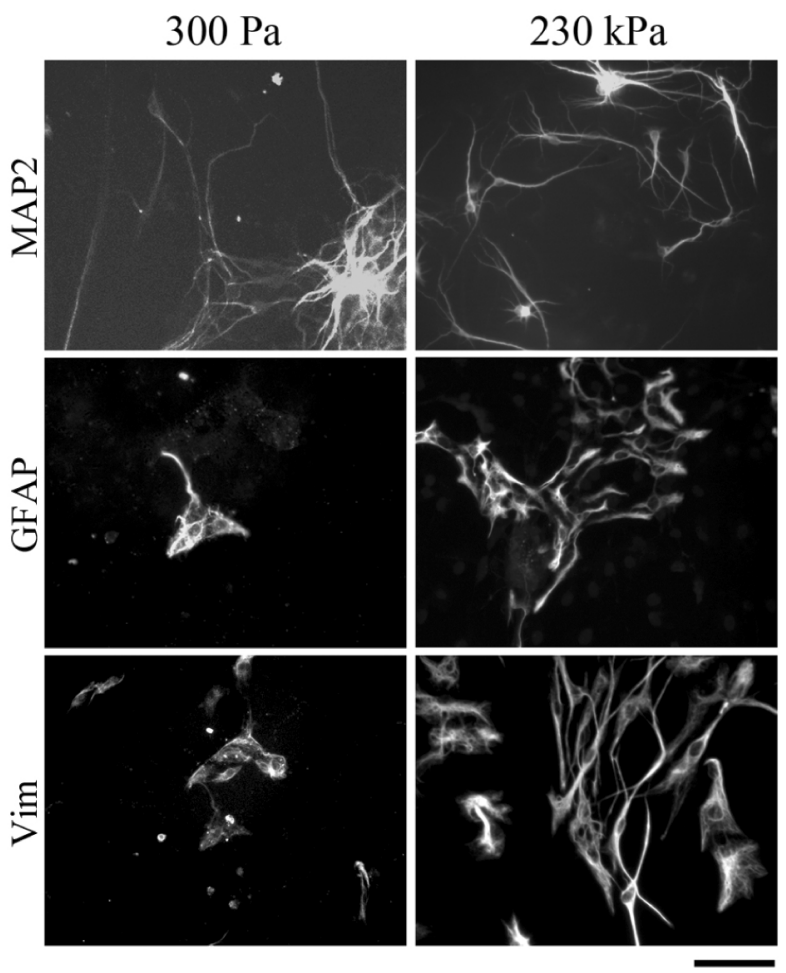

Fig. (3). Mixed cultures on PA gels. Top panel: MAP-2 immunocytochemistry is typical morphology of spinal cord neurons on 0.3 and $230 \mathrm{kPA}$ PA gels. Middle: Immuno for GFAP shows the growth of mature astrocytes on PA gels. Lower Panel: Immuno for vimentin (Vim) shows the growth of immature astrocytes. Scale bar: $100 \mu \mathrm{m}$.

Fig. (4) summarizes the alterations in number of neurons (A), mature (GFAP+) and immature (vimentin+) astrocytes ( $C$ and $\mathrm{B}$, respectively) along with neurite number (D) on gels with five different monomer concentrations. According

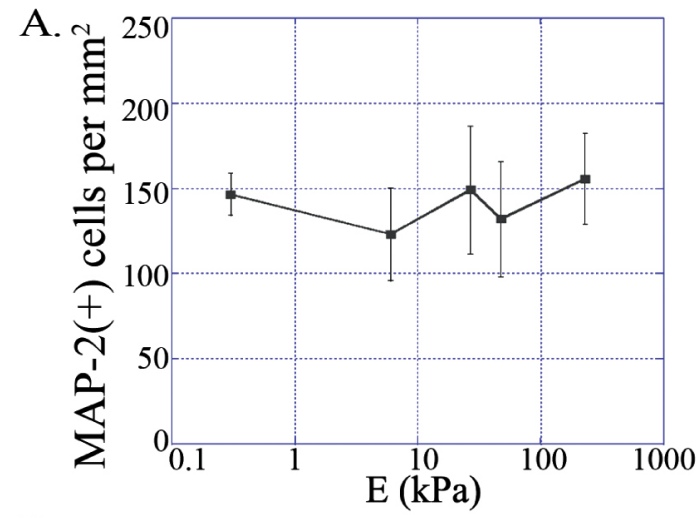

B.
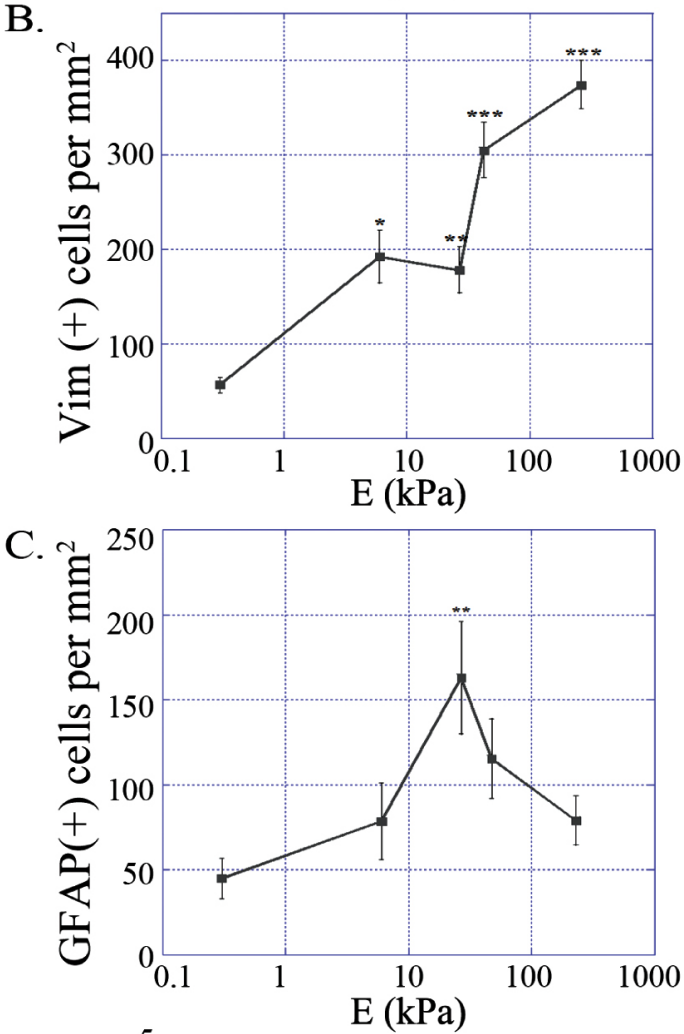

D.

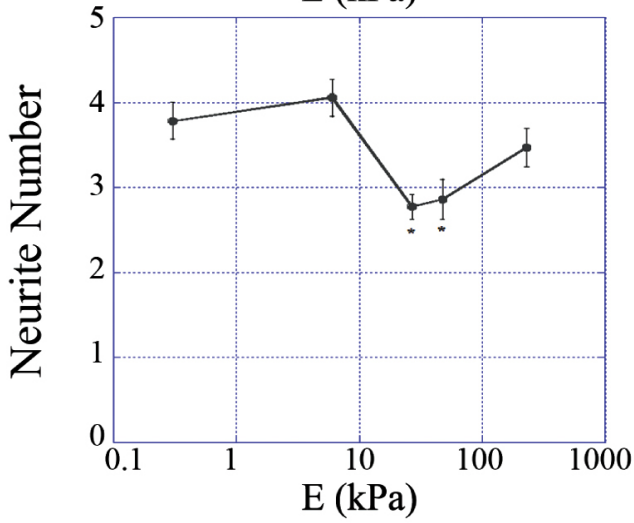

Fig. (4). Quantification of MAP-2 (A), GFAP (B), Vimentin (C) and primary neurite number on PA gels. *: $\mathrm{p}<0.05, * *: \mathrm{p}<0.01$, ***: $\mathrm{p}<0.001$ by ANOVA followed by Tukey's test for multiple comparisons versus $300 \mathrm{~Pa}$ gel. 
to MAP2 immunostaining used to specifically visualize neurons, we found that neuronal adhesion in mixed cultures does not significantly change as gel stiffness increases (Fig. 4A). The number of neurons on gels ranging in stiffness from 300 $\mathrm{Pa}$ to $230 \mathrm{kPa}$ was not significantly different. Immature astroglial cells (indicated by positive staining for vimentin, but not GFAP) increased in number as gel stiffness increased (Fig. 4B). All stiffnesses greater than $300 \mathrm{~Pa}$ had significantly more adherent immature astroglia $\left(\mathrm{p}_{6 \mathrm{kPa}}=0.04, \mathrm{p}_{27 \mathrm{kPa}}\right.$ $\left.=0.02, \mathrm{p}_{47 \mathrm{kPa}}<0.001, \mathrm{p}_{230 \mathrm{kPa}}<0.001\right)$. On the other hand, the response of mature astrocytes (visualized with GFAP immunostaining) to this range of stiffnesses exhibits a somewhat biphasic trend.

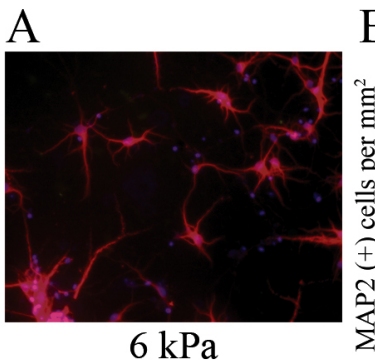

$6 \mathrm{kPa}$

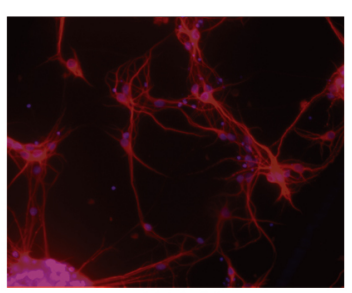

$47 \mathrm{kPa}$

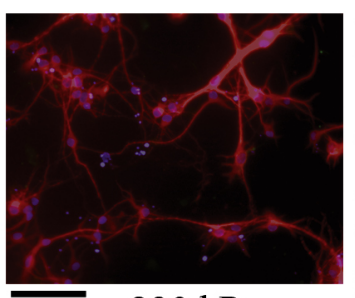

$230 \mathrm{kPa}$

MAP2 DDAPI $\square$ GFAP
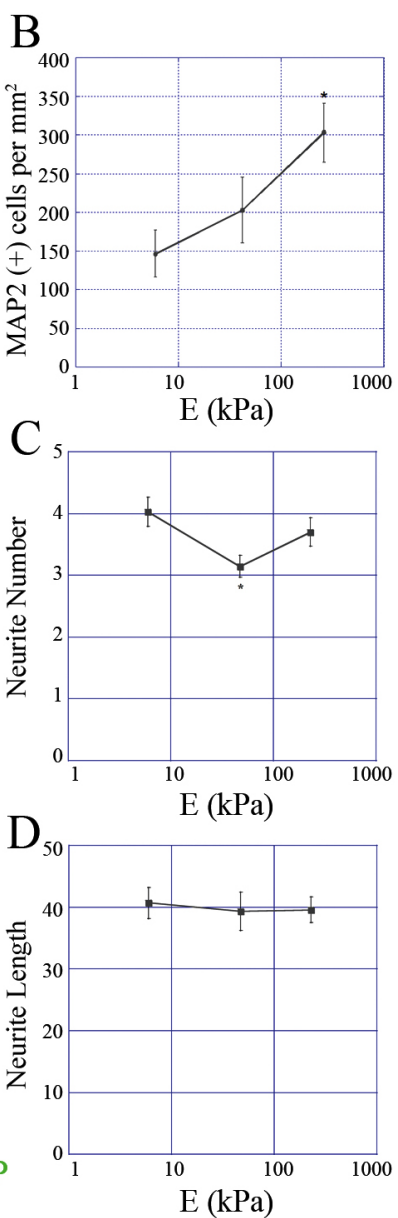

Fig. (5). Pure neurons on PA gels differ from mixed cultures. (A) MAP-2 (+) cell number increases (B) Neurite number, but not length differs with stiffness. Scale bar: $100 \mu \mathrm{m}$

Mature astroglia are least adhesive to softest (300 Pa) gels. There was a significantly higher number of adherent astrocytes on intermediate $27 \mathrm{kPa}$ gels compared to $300 \mathrm{~Pa}$ gels $(\mathrm{p}<0.01)$. Furthermore, while the average number of cells on $230 \mathrm{kPa}$ gels was lower than intermediate stiffnesses, these differences were not significant (Fig. 4C). These data suggest a possible optimal range of stiffness that best supports mature astrocyte adhesion and/or differentiation. Total cell number, determined by nuclear staining, increased with gel stiffness, though not significantly (data not shown). In addition to cell counting, we also examined neu- rite length and primary dendrite numbers in MAP2+ neurons. We found that there was no significant difference in neurite length with increasing stiffness (data not shown), with an average length of about $50 \mu \mathrm{m}$. Neurite number, however, was at a minimum when cells were plated on intermediate stiffnesses in mixed culture (Fig. 4D). Neurite number was significantly lower on $27 \mathrm{kPa}(\mathrm{p}<0.001)$ and 47 $\mathrm{kPa}(\mathrm{p}=0.04)$ gels than on $300 \mathrm{~Pa}$ gels.

A.
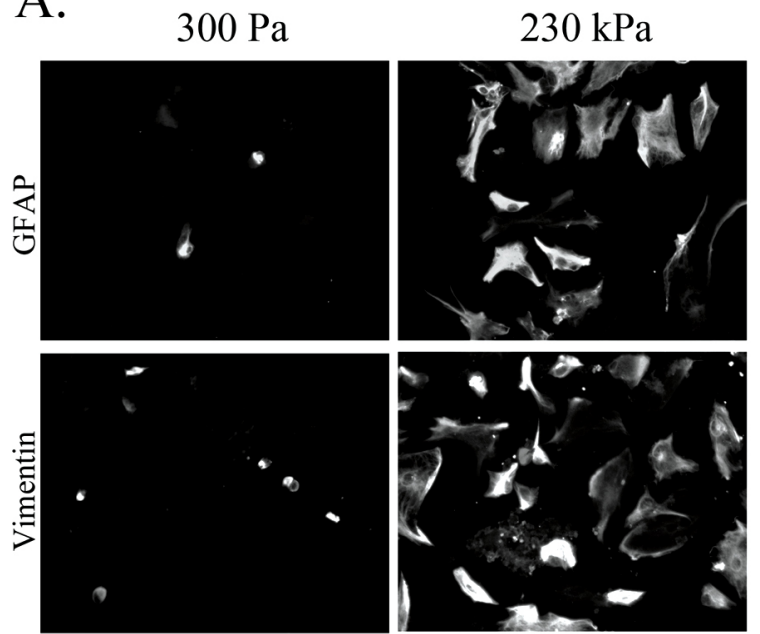

B.

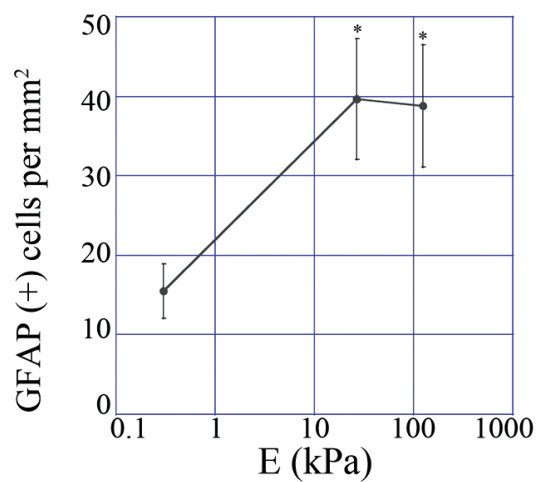

C.

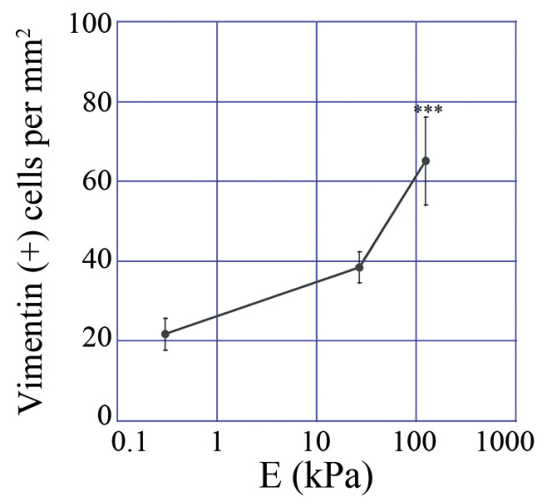

Fig. (6). Pure glia on PA gels. (A) Pure glia cultured on $300 \& 230$ $\mathrm{kPa}$ gels immunostained for GFAP or vimentin. GFAP+ (B) and Vimentin+ $(\mathbf{C})$ cell adhesion quantification. $* \mathrm{p}<0.05,{ }^{*} * \mathrm{p}<0.001$ by ANOVA followed by Tukey's test for multiple comparisons versus $0.3 \mathrm{kPa}$ gel.

\section{Effect of Gel Stiffness on Pure Neurons}

Based on GFAP, MAP2, and nuclear staining, we confirmed that $95 \%$ of the total cell population is neuronal. 
MAP2 (red) and GFAP (green) immunostaining show that neurons, but not astrocytes, grow in cultures (Fig. 5A). Typical neuronal morphology can be seen on all gel samples. Unlike their counterparts in the mixed culture, there are more spinal cord neurons in pure culture as the substrate becomes more rigid. There is a significant increase in MAP2 positive neuron numbers as gel stiffness increases from 6 to $230 \mathrm{kPa}$ $\left({ }^{*} p<0.05\right.$ by ANOVA followed by Tukey HSD test versus 6 kPa gel; Fig. 5B). In pure cultures, primary neurite number was examined on three of the five stiffnesses. Stiffnesses were chosen based on experimental data from mixed cultures, including one stiffness which yielded significantly fewer dendrites $(47 \mathrm{kPa})$ as well as two others with nonsignificant lower $(6 \mathrm{kPa})$ and higher $(230 \mathrm{kPa})$ dendrite numbers (Fig. 5C; ${ }^{*} \mathrm{p}<0.05$ by ANOVA followed by Tukey HSD test for multiple comparisons versus $6 \mathrm{kPa}$ gel.) Examination of neurite length revealed no significant difference among gel groups (Fig. 5D).
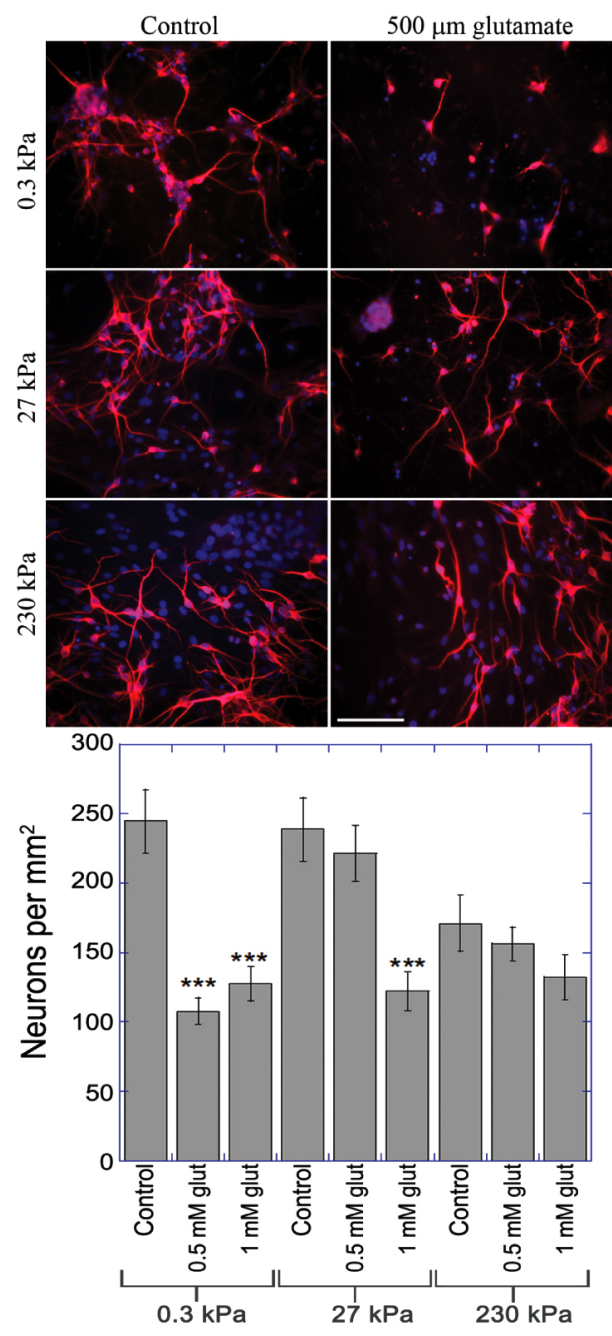

Fig. (7). Glutamate toxicity in mixed spinal cord neuron cultures. Control cultures and cells treated with $500 \mu \mathrm{M}$ glutamate are illustrated. Numbers of neurons that survived (MAP-2) were counted. Increasing concentrations of glutamate resulted in a more significant loss of spinal cord neurons. Results were derived from five independent experiments. $* * * p<0.001$ by ANOVA followed by Tukey's test for multiple comparisons against control groups. Scale bar, $100 \mu \mathrm{m}$.

\section{Effect of Gel Stiffness on Pure Astroglia Culture}

Representative images of pure astroglia immunostained for either GFAP or vimentin on two of the five stiffnesses tested are shown in Fig. (6A). GFAP+ cell adhesion in pure cultures was very low on 300 Pa gels, and cells preferentially adhered to higher stiffnesses (Fig. 6B). Unlike mixed cultures, we did not observe maximal adhesion at intermediate stiffness but rather a plateau of the adhesion curve on stiffnesses above $27 \mathrm{kPa}$. Vimentin+ cell adhesion in pure culture increased with stiffness (Fig. 6C), similar to the adhesion trend observed for immature astroglia in mixed cultures (Fig. 4B).

\section{Matrix Stiffness Affects Glutamate-Induced Neuro- toxicity}

To examine the survival of spinal cord neurons on PA gels of different stiffness, we treated mixed spinal cord cultures with glutamate at a concentration of either $500 \mu \mathrm{M}$ or $1 \mathrm{mM}$. Cells were treated for 1 hour at DIV6, the glutamate solution was removed, and 24 hours later, the cultures were fixed and immunostained for MAP2 and DAPI. Surviving neurons on all stiffnesses appear healthy and bear multiple processes (Fig. 7, top). Glutamate-elicited neuronal cell death increased in a dose-dependent manner on $27 \mathrm{kPa}$ and $230 \mathrm{kPa}$ gels, with a significant level of toxicity only seen on $27 \mathrm{kPa}$ gels with cells treated with $1 \mathrm{mM}$ glutamate. On 300 Pa gels, however, $500 \mu \mathrm{M}$ glutamate achieved the same toxicity level as $1 \mathrm{mM}$ (Fig 7, bottom). After treatment with a glutamate concentration of $500 \mu \mathrm{M}$, approximately $40 \%$ of the neurons did not survive on soft gels (Fig. 8). On harder gels, exposure to $500 \mu \mathrm{M}$ glutamate at DIV6 did not result in a significant reduction in cell number.

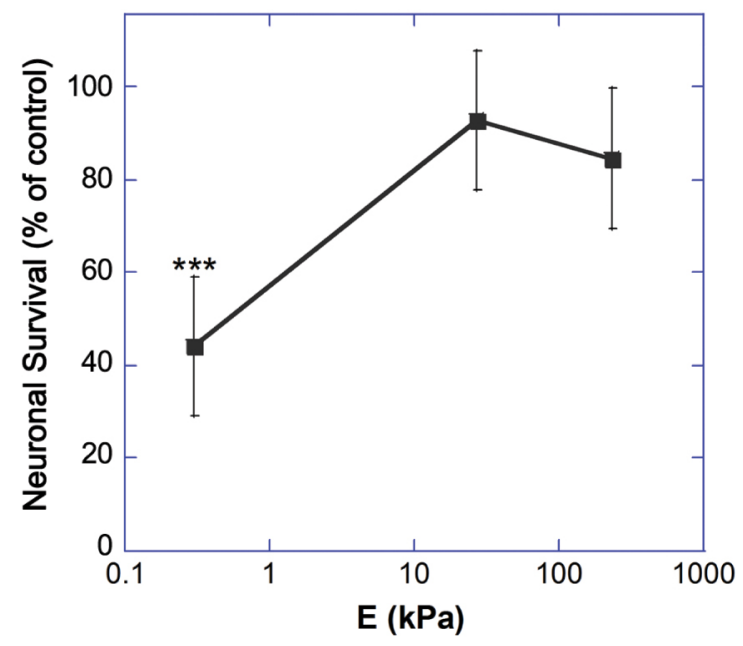

Fig. (8). Population of neurons surviving glutamate treatment compared to control cultures. ***p $<0.001$ by ANOVA followed by Tukey's test for multiple comparisons against $0.3 \mathrm{kPa}$ gels.

\section{DISCUSSION}

Our current work asks whether or not differences in neuronal behavior induced by substrate rigidity can be fully attributed to mechanosensing of neurons alone or whether interactions between neurons and glial cells can affect their respective responses to mechanical stimuli. We employed a 
well-developed polyacrylamide gel system, and by exposing cells to substrates with different mechanical stiffnesses but identical surface adhesion properties, we evaluated cell number, neurite length, and primary neurite number, either in coculture or in pure cultures. Neuronal adhesion increased with increasing elastic modulus of the substrate in pure, but not mixed, cultures (Figs. 4A and 5B). Interactions between neurons and astroglia may contribute to this difference. It is possible that there is competition for binding sites on the gel surface or that there is a trophic influence on neurons imparted by the astrocytes that are present in mixed cultures. The differences in neuronal number in mixed cultures compared to pure cultures may have also occurred due to proliferation of neuronal precursors, a variable that would be removed when cells were treated with mitotic inhibitors in pure neuronal cultures. The precursor cells are present in the mixed culture at the time of plating and will proliferate for several days [23], a variable that may indeed be influenced by the mechanics of the substrate, a hypothesis supported by the finding that mesenchymal stem cells preferentially differentiate into neuronal phenotypes on soft gels [24]. Finally, neurons are often found growing in aggregates with astrocytes and it is possible that neurons are adhering to astrocytes and are stimulated by their mechanical stiffness, which has been reported to be approximately $300-500 \mathrm{~Pa}$ [25], rather than that of the underlying substrate.

We have also found that the adhesion response of mature GFAP+ astrocytes to the changes in gel stiffness was different in co-culture (Fig. 4C) than in pure astroglial culture (Fig. 6B). In the presence of neurons, adhesion number peaked at intermediate stiffness and cells on hardest gels were not significantly more adherent than on softer gels. In pure cultures, however, the adhesion value was significantly higher on both intermediate stiffness and hardest gels tested, a difference that is potentially also contributed to cell competition for binding sites in mixed cultures. Finally, vimentin+ cell adhesion increased with increasing stiffness, possibly indicating that immature, proliferative, differentiating cells preferentially adhere to harder substrates both alone or in the presence of other cell types (Figs 4B and 6C).

The differential effect of the mechanical characteristics of the hydrogel materials was observed in co-culture of neurons and astroglia (Figs. 4A and $\mathbf{4 C}$ ). These data will aid in the development of biomaterial scaffold designs, which could potentially support and promote neuronal growth while inhibiting astrocyte proliferation, differentiation, or growth. This is important for inhibition of a glial scar when regeneration of neurons after spinal cord injury is an issue. Previous studies of mouse spinal cord neurons suggest a preference of neuronal cells to grow on softer gels based on a marked increase in branching number [9]. We found that neuronal number does not differ significantly in the mixed culture on the range of stiffnesses under study. Glial cells were also observed on the softest gels in our study, which is different than the previous report [9]. Like previous studies, we have found that there are proportionally more neurons on soft gels in mixed culture, possibly due to inhibition of astroglial attachment [10].

Glutamate is a major neurotransmitter of the spinal cord. Astrocytes function to clear extracellular glutamate, presumably preventing neuronal toxicity. Astrocytes are neces- sary in culture to prevent glutamate toxicity [27-29]. In our PA gel system in which small changes can alter the resulting population of astrocytes in a mixed culture, we also observe variations in neuronal resistance to glutamate. Neurons are most resistant to $500 \mu \mathrm{M}$ glutamate treatment in co-cultures where a high proportion of astrocytes are present (Figs. 7 and 8). Furthermore, increasing glutamate levels to $1 \mathrm{mM}$ results in a similar number of adherent neurons in all cultures, perhaps indicating that there is a subpopulation of immature neurons in all cultures that are resistant to glutamateinduced toxicity at DIV6 (Fig. 7). Our results conclude that neurons are somewhat dependent on astrocytes in a coculture system for functional protective effects, though not for cell adhesion and morphological development.

There will certainly be differences regarding how neurons respond to mechanical changes in vivo versus in vitro. Cells in vivo are surrounded by extracellular matrix, a network of semi-flexible polymers; semi-flexible polymers stiffen under strain, which would occur when neurons and astrocytes pull on their matrix via myosin motors at focal adhesion sites. Polyacrylamide gels, on the other hand, are nearly ideally elastic and mechanical properties are stable under strain. Furthermore, the three dimensional component provides that focal receptors on both the basal and ventral side of the cell are engaged, which may lower the cell's threshold for mechanical inputs.

Finally, although the mechanical characterization of bisgels has also been carried out by other investigators [8-12, 26]; Table 1), there are a couple of distinctions in our study. First, a non-intrusive method was used to measure the stiffness in situ. Second, other investigators altered the stiffness of the gels by keeping monomer concentration constant while varying crosslink concentration. In our study, monomer/crosslink ratio remained constant and the amounts of both were changed simultaneously. Third, we examined gels with broader range of stiffnesses $(300 \mathrm{~Pa} 230 \mathrm{kPa})$ than those used in other studies $(150 \mathrm{~Pa} \sim 1600 \mathrm{~Pa}$ in [9] and 600 $\mathrm{Pa} \sim 27 \mathrm{kPa}$ in [10]). We chose gel stiffnesses at these different magnitudes in such a way that we span the range of natural CNS material (300 Pa) to the stiffest gel (20\%), which is close to the stiffness reported for implant materials. This selection is based on the possibility that the physiological stiffness condition is not necessarily the optimal condition for spinal cord neuron regeneration in vitro. Fourth, the tissue type (cortex vs. spinal cord) could contribute to differences as well as culture medium conditions, for which we used serum-free medium.

In summary, our study not only confirms that the mechanical stiffness of the substrate impacts neuronal and astroglial survival and growth, but it also reveals that the way these individual cell types respond to mechanical stimuli are altered in the presence and absence of interaction with one another. The results from this study provide important information for our understanding of the mechanical aspects of cellular-ECM interactions. Further studies must be carried out before we can translate our in vitro data to developing cures for traumatic CNS injuries.

\section{ACKNOWLEDGEMENTS}

This study was supported by grants from the New Jersey Commission on Spinal Cord Research (Grant \# 05-3041- 
SCR-E-0 to N.A.L. and B.L.F. and Grant \# 03-3024-SCR-E0 to B.L.F.). M.L.P is a graduate trainee of the IGERT Program on Integratively Engineered Biointerfaces at Rutgers: NSF Grant DGE-0333196. M.K.K. is a graduate trainee of the Rutgers-UMDNJ Biotechnology Training Program: National Institute of General Medical Sciences 5T32GM008339-17. The authors also thank Dr. David Shreiber, Dr. Rene Schloss, Dr. Bernard Yurke, and Dr. David Lin for constructive discussions and advice. Uday Chippada's assistance in mechanical testing of the gels is appreciated.

\section{REFERENCES}

[1] Geiger B. Cell biology. Encounters in space. Science 2001; 294(5547):1661-3.

[2] Griffith LG, Swartz MA. Capturing complex 3D tissue physiology in vitro. Nat Rev Mol Cell Biol 2006; 7(3): 211-24.

[3] Stegemann JP, Hong H, Nerem RM. Mechanical, biochemical, and extracellular matrix effects on vascular smooth muscle cell phenotype. J Appl Physiol 2005; 98(6): 2321-7.

[4] Curtis A, Riehle M. Tissue engineering: the biophysical background. Phys Med Biol 2001; 46(4): R47-65.

[5] Khatiwala CB, Peyton SR, Putnam AJ. Intrinsic mechanical properties of the extracellular matrix affect the behavior of preosteoblastic MC3T3-E1 cells. Am J Physiol Cell Physiol 2006; 290(6): C1640-50.

[6] Pedersen JA, Swartz MA. Mechanobiology in the third dimension. Ann Biomed Eng 2005; 33(11): 1469-90.

[7] Georges PC, Janmey PA. Cell type-specific response to growth on soft materials. J Appl Physiol 2005; 98(4): 1547-53.

[8] Wang YL, Pelham RJ, Jr. Preparation of a flexible, porous polyacrylamide substrate for mechanical studies of cultured cells. Methods Enzymol 1998; 298: 489-96.

[9] Flanagan LA, Ju YE, Marg B, Osterfield M, Janmey PA. Neurite branching on deformable substrates. Neuroreport 2002; 13(18): 2411-5.

[10] Georges PC, Miller WJ, Meaney DF, Sawyer ES, Janmey PA. Matrices with compliance comparable to that of brain tissue select neuronal over glial growth in mixed cortical cultures. Biophys $\mathbf{J}$ 2006; 90(8): 3012-8.

[11] Pelham RJ, Wang Y-L. Cell locomotion and focal adhesions are regulated by substrate flexibility. Proc Natl Acad Sci USA 1997; 94: 13661-5.

[12] Wong JY, Velasco A, Rajagopalan P, Pham Q. Directed movement of vascular smooth muscle cells on gradient-compliant hydrogels. Langmuir 2003; 19(5): 1908-13.

[13] Semler EJ, Ranucci CS, Moghe PV. Tissue assembly guided via substrate biophysics: applications to hepatocellular engineering. Adv Biochem Eng Biotechnol 2006; 102: 1-46.
[14] Gefen A, Gefen N, Zhu Q, Raghupathi R, Margulies SS. Agedependent changes in material properties of the brain and braincase of the rat. J Neurotrauma. 2003; 20(11): 1163-77.

[15] Hirakawa K, Hashizume K, Hayashi T. Viscoelastic property of human brain -for the analysis of impact injury (author's transl). No To Shinkei. 1981; 33(10): 1057-65.

[16] Miller K, Chinzei K, Orssengo G, Bednarz P. Mechanical properties of brain tissue in-vivo: experiment and computer simulation. J Biomech 2000; 33(11): 1369-76.

[17] Dalton PD, Flynn L, Shoichet MS. Manufacture of poly(2hydroxyethyl methacrylate-co-methyl methacrylate) hydrogel tubes for use as nerve guidance channels. Biomaterials 2002; 23(18): 3843-51.

[18] Teng YD, Lavik EB, Qu X, Park KI, Ourednik J, Zurakowski D, et $a l$. Functional recovery following traumatic spinal cord injury mediated by a unique polymer scaffold seeded with neural stem cells. Proc Natl Acad Sci USA 2002; 99(5): 3024-9.

[19] Lin DC, Yurke B, Langrana NA. Mechanical properties of a reversible, DNA-crosslinked polyacrylamide hydrogel. J Biomech Eng 2004; 126(1): 104-10.

[20] Lin DC, Yurke B, Langrana NA. Use of rigid spherical inclusions in Young's moduli determination: application to DNA-crosslinked gels. J Biomech Eng 2005; 127(4): 571-9.

[21] McCarthy KD, de Vellis J. Preparation of separate astroglial and oligodendroglial cell cultures from rat cerebral tissue. J Cell Biol 1980; 85(3): 890-902.

[22] Akum BF, Chen M, Gunderson SI, Riefler GM, Scerri-Hansen MM, Firestein BL. Cypin regulates dendrite patterning in hippocampal neurons by promoting microtubule assembly. Nat Neurosci 2004; 7(2): 145-52.

[23] Deloulme JC, Baudier J, Sensenbrenner M. Establishment of pure neuronal cultures from fetal rat spinal cord and proliferation of the neuronal precursor cells in the presence of fibroblast growth factor. J Neurosci Res 1991; 29(4): 499-509.

[24] Engler AJ, Sen S, Sweeney HL, Discher DE. Matrix elasticity directs stem cell lineage specification. Cell 2006; 126(4): 677-89.

[25] Lu YB, Franze K, Seifert G, Steinhauser C, Kirchhoff F, Wolburg $\mathrm{H}$, et al. Viscoelastic properties of individual glial cells and neurons in the CNS. Proc Natl Acad Sci USA 2006; 103(47): 1775964.

[26] Engler A, Bacakova L, Newman C, Hategan A, Griffin M, Discher D. Substrate compliance versus ligand density in cell on gel responses. Biophys J 2004; 86(1): 617-28.

[27] Brown DR Neurons depend on astrocytes in a coculture system for protection from glutamate toxicity. Mol Cell Neurosci 1999; 13(5): 379-89.

[28] Rosenberg PA, Aizenman E. Hundred-fold increase in neuronal vulnerability to glutamate toxicity in astrocyte-poor cultures of rat cerebral cortex. Neurosci Lett 1989; 103(2): 162-8.

[29] Rosenberg PA, Amin S, Leitner M. Glutamate uptake disguises neurotoxic potency of glutamate agonists in cerebral cortex in dissociated cell culture. J Neurosci 1992; 12(1): 56-61. 